\title{
Effects of backreaction on power-Maxwell holographic superconductors in Gauss-Bonnet gravity
}

\author{
Hamid Reza Salahi ${ }^{1, \mathrm{a}}$, Ahmad Sheykhi ${ }^{1,2, \mathrm{~b}}$, Afshin Montakhab ${ }^{1, \mathrm{c}}$ \\ ${ }^{1}$ Physics Department and Biruni Observatory, College of Sciences, Shiraz University, Shiraz 71454, Iran \\ ${ }^{2}$ Research Institute for Astronomy and Astrophysics of Maragha (RIAAM), P.O. Box 55134-441, Maragha, Iran
}

Received: 2 August 2016 / Accepted: 14 October 2016 / Published online: 25 October 2016

(C) The Author(s) 2016. This article is published with open access at Springerlink.com

\begin{abstract}
We analytically and numerically investigate the properties of $s$-wave holographic superconductors by considering the effects of scalar and gauge fields on the background geometry in five-dimensional Einstein-Gauss-Bonnet gravity. We assume the gauge field to be in the form of the powerMaxwell nonlinear electrodynamics. We employ the SturmLiouville eigenvalue problem for analytical calculation of the critical temperature and the shooting method for the numerical investigation. Our numerical and analytical results indicate that higher curvature corrections affect condensation of the holographic superconductors with backreaction. We observe that the backreaction can decrease the critical temperature of the holographic superconductors, while the power-Maxwell electrodynamics and Gauss-Bonnet coefficient term may increase the critical temperature of the holographic superconductors. We find that the critical exponent has the mean-field value $\beta=1 / 2$, regardless of the values of Gauss-Bonnet coefficient, backreaction and power-Maxwell parameters.
\end{abstract}

\section{Introduction}

In 2008, Hartnol et al., put forwarded a new step on the application of the gauge/gravity duality in condensedmatter physics $[1,2]$. They have claimed that some properties of strongly coupled superconductors can be potentially described by classical general relativity living in one higher dimension. This novel idea is usually called holographic superconductors. The motivation is to shed light on the understanding the mechanism governing the hightemperature superconductors in condensed-matter physics.

\footnotetext{
a e-mail: hrsalahi@shirazu.ac.ir

be-mail: asheykhi@shirazu.ac.ir

c e-mail: montakhab@shirazu.ac.ir
}

The holographic $s$-wave superconductor model known as Abelian-Higgs model was first established in [1,2]. The wellknown duality between anti-de Sitter (AdS) spacetime and the conformal field theories (CFT) [3-5] implies that there is a correspondence between the gravity in the $d$-dimensional spacetime and the gauge field theory livening on its $(d-1)$ dimensional boundary. According to the idea of the holographic superconductors given in [1], in the gravity side, a Maxwell field and a charged scalar field are introduced to describe the $U(1)$ symmetry and the scalar operator in the dual field theory, respectively. This holographic model undergoes a phase transition from black hole with no hair (normal phase/conductor phase) to the case with scalar hair at low temperatures (superconducting phase) [6].

Following [1,2], an overwhelming number of papers have appeared which try to investigate various properties of the holographic superconductors from different perspective [7-16]. The studies were also generalized to other gravity theories. In the context of Gauss-Bonnet gravity, the phase transition of the holographic superconductors were explored in [17-23]. The motivation is to study the effects of higher order gravity corrections on the critical temperature of the holographic superconductors. Considering the holographic $p$-wave and $s$-wave superconductors in $(3+1)$ dimensional boundary field theories, it was shown that when Gauss-Bonnet coefficients become larger the operators on the boundary field theory will be harder to condense [19]. Taking the backreaction of the gauge and scalar field on the background geometry into account, a numerical as well as an analytical study of the holographic superconductors in five-dimensional Einstein-Gauss-Bonnet gravity were carried out in [20]. It was observed that the temperature of the superconductor decreases with increasing backreaction, although the effect of the Gauss-Bonnet coupling is more subtle: the critical temperature first decreases, then increases as the coupling tends toward the Chern-Simons value in a backreaction dependent fashion [20]. 
In addition to the correction on the gravity side of the action, it is also interesting to consider the corrections to the gauge field on the matter side of the action. In particular, it is interesting to investigate the effects of the nonlinear corrections to the gauge field on the condensation and the critical temperature of the holographic superconductors. It was argued that in the Schwarzschild AdS black hole background, the higher nonlinear electrodynamics corrections make the condensation harder [24-27]. When the gauge field is in the form of Born-Infeld nonlinear electrodynamics, an analytical study, based on the Sturm-Liouville eigenvalue problem, of holographic superconductors in Einstein [28-33] and Gauss-Bonnet gravity [34-37] has been carried out. In the background of $d$-dimensional Schwarzschild AdS black hole, the properties of power-Maxwell holographic superconductors have been explored in the probe limit [38] and away from the probe limit [39]. In our recent paper [40], we have analytically as well as a numerically studied the holographic $s$-wave superconductors in Gauss-Bonnet gravity with power-Maxwell electrodynamics. However, in that work, we did not investigate the effects of backreaction and limited our study to the case where scalar and gauge fields do not have an effect on the background metric. Our purpose in the present work is to disclose the effects of the backreaction on the phase transition and the critical temperature of the power-Maxwell holographic superconductors in GaussBonnet gravity.

The organization of this paper is as follows. In the next section, we provide the basic field equations of power-Maxwell holographic superconductors in the background of GaussBonnet-AdS black holes by taking into account the backreaction. In Sect. 3, based on the Sturm-Liouville eigenvalue problem, we find a relation between the critical temperature and the charge density of the backreacting holographic superconductor with Maxwell field in Gauss-Bonnet gravity. In Sect. 4, we extend the study to the case of powerMaxwell nonlinear electrodynamics. By applying the shooting method, we also compare our analytical calculations with numerical results in this section. In Sect. 5, we calculate the critical exponent and the condensation values of the power-Maxwell holographic superconductor with backreaction. We finish with our conclusion and a discussion in Sect. 6.

\section{Backreacting Gauss-Bonnet holographic superconductors}

To study a $(3+1)$-dimensional holographic superconductor, we begin with a $(4+1)$-dimensional action of EinsteinGauss-Bonnet-AdS gravity which is coupled to powerMaxwell field and a charged scalar field,

$$
\begin{aligned}
S= & \int \mathrm{d}^{5} x \sqrt{-g} \frac{1}{2 \kappa^{2}}\left[(R-2 \Lambda)+\frac{\alpha}{2}\left(R^{2}-4 R^{\mu \nu} R_{\mu \nu}\right.\right. \\
& \left.+R^{\mu \nu \rho \sigma} R_{\mu \nu \rho \sigma}\right]+\int \mathrm{d}^{5} x \sqrt{-g} \\
& \times\left[-b\left(F_{\mu \nu} F^{\mu \nu}\right)^{q}-|\nabla \psi-i e A \psi|^{2}-m^{2}|\psi|^{2}\right],
\end{aligned}
$$

where $\kappa^{2}=8 \pi G_{5}$ with $G_{5}$ is the five-dimensional gravitational constant, $\Lambda=-6 / l^{2}$ is the negative cosmological constant, where $l$ is the AdS radius of spacetime, and $\alpha$ is the Gauss-Bonnet coefficient. Here, $R$ and $R_{\mu \nu}$ and $R_{\mu \nu \sigma \rho}$ are, respectively, the Ricci scalar, the Ricci tensor and Riemann curvature tensor. $F^{\mu \nu}$ is the electromagnetic field tensor and $q$ is the power parameter of the power-Maxwell field. $\psi$ is a complex scalar field with the charge $e$ and the mass $m$, and $A$ is the gauge field. Also, $b$ is coupling constant and due to positivity of energy density has sign $(-1)^{q+1}[41,42]$. For latter convenience we shall take $b=(-1 / 2)^{q+1}$. With this choice, the power-Maxwell Lagrangian will reduce to the Maxwell Lagrangian in the limit $q=1$.

It is easy to check that by re-scaling $\psi \rightarrow \tilde{\psi} / e, \phi \rightarrow \tilde{\phi} / e$ and $b \rightarrow \tilde{b} e^{2 q-2}$, a factor $1 / e^{2}$ will appear in front of matter part of action (1). Thus, the probe limit can be deduced when $\kappa^{2} / e^{2} \rightarrow 0$. In order to take the backreaction into account, in this paper, we keep $\kappa^{2} / e^{2}$ finite and for simplicity we set $e$ as unity.

Taking the backreaction into account, the plane-symmetric black hole solution with an asymptotically AdS behavior in five-dimensional spacetime may be written

$\mathrm{d} s^{2}=-e^{-\chi(r)} f(r) \mathrm{d} t^{2}+\frac{\mathrm{d} r^{2}}{f(r)}+\frac{r^{2}}{l_{\text {eff }}^{2}}\left(\mathrm{~d} x^{2}+\mathrm{d} y^{2}+\mathrm{d} z^{2}\right)$,

where

$l_{\text {eff }}^{2} \equiv \frac{2 \alpha}{1-\sqrt{1-\frac{4 \alpha}{l^{2}}}}$,

is the effective AdS radius of the spacetime. The ratio of $l_{\text {eff }} / l$ can be smaller than unity for $\alpha>0$, while for $\alpha<0$ it is obvious that $l_{\mathrm{eff}} / l$ is larger than unity.

The superconductivity phase transition is dual to formation of charged matter field in the bulk, and for occurrence this phase transition in bulk, one needs to prevent the charged matter field to falls into the black hole, thus we expect greater curvature of spacetime in bulk make condensation harder which corresponds to the positive values of $\alpha$. Also for $\alpha<0$, we shall see that the scalar field can be formed easier, means at higher temperature.

The Hawking temperature of black hole is given by

$T=\frac{f^{\prime}\left(r_{+}\right) e^{-\chi(r+) / 2}}{4 \pi}$,

where $r_{+}$is the black hole horizon and the prime denotes derivative with respect to $r$. We choose the electromagnetic 
gauge potential and scalar field as

$\psi=\psi(r), \quad A=\phi(r) \mathrm{d} t$.

Without loss of generality, we can take $\phi(r)$ and $\psi(r)$ real. The equation of motions can be obtained by varying action (1) with respect to the metric and matter fields. We find

$$
\begin{aligned}
& \psi^{\prime \prime}+\psi^{\prime}\left(\frac{f^{\prime}}{f}+\frac{3}{r}-\frac{\chi^{\prime}}{2}\right)+\psi\left(\frac{\phi^{2}}{f^{2}}-\frac{m^{2}}{f}\right)=0 \\
& \phi^{\prime \prime}+\phi^{\prime}\left(\frac{3}{(2 q-1) r}+\frac{\chi^{\prime}}{2}\right)-\frac{2 e^{(1-q) \chi} \psi^{2} \phi^{\prime 2-2 q}}{q(2 q-1) f} \phi=0 \\
& \chi^{\prime}\left(1-\frac{2 \alpha f}{r^{2}}\right)+\frac{4 r \kappa^{2}}{3}\left(\psi^{\prime 2}+\frac{e^{\chi} \phi^{2} \psi^{2}}{f^{2}}\right)=0 \\
& f^{\prime}\left(1-\frac{2 \alpha f}{r^{2}}\right)+\frac{2 f}{r}-\frac{4 r}{l^{2}}+\frac{2 r \kappa^{2}}{3} \\
& \quad \times\left(m^{2} \psi^{2}+f \psi^{\prime 2}+\frac{e^{\chi} \phi^{2} \psi^{2}}{f}+\frac{(2 q-1)}{2} e^{q \chi} \phi^{\prime 2 q}\right)=0 .
\end{aligned}
$$

In order to solve the above field equations, we need appropriate boundary conditions both on the horizon $r_{+}$, which is defined by $f\left(r_{+}\right)=0$, and on the AdS boundary where $r \rightarrow \infty$. On the horizon, the regularity condition imposes

$\phi\left(r_{+}\right)=0, \quad \psi^{\prime}\left(r_{+}\right)=\frac{m^{2} \psi\left(r_{+}\right)}{f^{\prime}\left(r_{+}\right)}$,

and thus from Eqs. (8) and (9) we have

$$
\begin{aligned}
\chi^{\prime}\left(r_{+}\right)= & -\frac{4 \kappa^{2} r_{+}}{3}\left(\psi^{\prime}\left(r_{+}\right)^{2}+\frac{e^{\chi\left(r_{+}\right)} \phi^{\prime}\left(r_{+}\right)^{2} \psi\left(r_{+}\right)^{2}}{f^{\prime}\left(r_{+}\right)^{2}}\right), \\
f^{\prime}\left(r_{+}\right)= & \frac{4 r_{+}}{l^{2}}-\frac{2 \kappa^{2} r_{+}}{3} \\
& \times\left(m^{2} \psi\left(r_{+}\right)^{2}+\frac{(2 q-1)}{2} e^{q \chi\left(r_{+}\right)} \phi^{\prime}\left(r_{+}\right)^{2 q}\right) .
\end{aligned}
$$

Since our solutions are asymptotically AdS, as $r \rightarrow \infty$, we have

$$
\begin{aligned}
& \chi(r) \rightarrow 0, \quad f(r) \approx \frac{r^{2}}{l_{\mathrm{eff}}^{2}}, \\
& \phi(r) \approx \mu-\frac{\rho^{\frac{1}{2 q-1}}}{r^{\frac{4-2 q}{2 q-1}}}, \quad \psi \approx \frac{\psi_{-}}{r^{\Delta_{-}}}+\frac{\psi_{+}}{r^{\Delta_{+}}},
\end{aligned}
$$

where $\mu$ and $\rho$ are, respectively, the chemical potential and charge density of the CFT boundary, and $\Delta_{ \pm}$is defined as

$$
\Delta_{ \pm}=2 \pm \sqrt{4+m^{2} l_{\mathrm{eff}}^{2}} \text {. }
$$

According to the AdS/CFT correspondence, $\psi_{ \pm}=<\mathcal{O}_{ \pm}>$, where $\mathcal{O}_{ \pm}$is the dual operator to the scalar field with the conformal dimension $\Delta_{ \pm}$. We have the freedom to impose boundary conditions such that either $\psi_{-}$or $\psi_{+}$vanish. We prefer to keep fixed $\Delta_{ \pm}$while we vary $\alpha$, thus we set $\tilde{m}^{2}=$ $m^{2} l_{\text {eff }}^{2}$. For example, for $\tilde{m}^{2}=-3$, we have $\Delta_{+}=3$ for all values of parameter $\alpha$.

It is important to note that, unlike other known versions of electrodynamics, the boundary condition for the gauge field $\phi(r)$ given in Eq. (13), depends on the power parameter $q$. Using boundary condition (13) and the fact that $\phi$ should be finite as $r \rightarrow \infty$, we require that $(4-2 q) /(2 q-1)>0$ which restricts $q$ to the range $1 / 2<q<2$.

It is easier to work in the dimensionless variable, $z=$ $r_{+} / r$, instead of variable $r$. Under this transformation, the equations of motion (6)-(9) become

$$
\begin{aligned}
& \psi^{\prime \prime}+\left(\frac{f^{\prime}}{f}-\frac{1}{z}-\frac{\chi^{\prime}}{2}\right) \psi^{\prime} \\
& +\frac{r_{+}^{2}}{z^{4}}\left(\frac{\phi^{2} e^{\chi}}{f^{2}}-\frac{m^{2}}{f}\right) \psi=0, \\
& \phi^{\prime \prime}+\left(\frac{4 q-5}{(2 q-1) z}+\frac{\chi^{\prime}}{2}\right) \phi^{\prime} \\
& -\frac{2 r_{+}^{2 q} \psi^{2} \phi^{\prime 2-2 q}}{(-1)^{2 q} q(2 q-1) z^{4 q} f} \phi=0,
\end{aligned}
$$

$\chi^{\prime}\left(1-\frac{2 \alpha z^{2} f}{r_{+}^{2}}\right)-\frac{4 \kappa^{2} r_{+}^{2}}{3 z^{3}}\left(\frac{e^{\chi} \psi^{2} \phi^{2}}{f^{2}}+\frac{z^{4} \psi^{\prime 2}}{r_{+}^{2}}\right)=0$

$$
\begin{aligned}
& f^{\prime}\left(1-\frac{2 \alpha z^{2} f}{r_{+}^{2}}\right)-\frac{2 f}{z}+\frac{4 r_{+}^{2}}{l^{2} z^{3}}-\frac{2 \kappa^{2} r_{+}^{2}}{3 z^{3}}\left[\frac{z^{4} f \psi^{\prime 2}}{r_{+}^{2}}\right. \\
& +\frac{e^{\chi} \psi^{2} \phi^{2}}{f}+m^{2} \psi^{2}-\frac{1}{2}(1-2 q) \\
& \left.\times e^{q \chi}(-1)^{2 q}\left(\frac{z^{2} \phi^{\prime}}{r_{+}}\right)^{2 q}\right]=0 .
\end{aligned}
$$

Here the prime indicates the derivative with respect to the new coordinate $z$ which ranges in the interval $[0,1]$, where $z=0$ and $z=1$ correspond to the boundary and horizon, respectively. Since near the critical point the expectation value of scalar operator $\left(<\mathcal{O}_{ \pm}>\right)$is small, we can select it as an expansion parameter

$\epsilon \equiv<\mathcal{O}_{i}>$,

where $i= \pm$. Using the fact that $\epsilon \ll$, we can expand $f$ and $\chi$ around the Gauss-Bonnet AdS spacetime as

$f=f_{0}+\epsilon^{2} f_{2}+\epsilon^{4} f_{4}+\cdots$,

$\chi=\epsilon^{2} \chi_{2}+\epsilon^{4} \chi_{4}+\cdots$ 
Note that since we are interested in solution in which condensation is small, $\psi$ and $\phi$ can also be expanded as

$\psi=\epsilon \psi_{1}+\epsilon^{3} \psi_{3}+\epsilon^{5} \psi_{5}+\cdots$,

$\phi=\phi_{0}+\epsilon^{2} \phi_{2}+\epsilon^{4} \phi_{4}+\cdots$

We further assume the chemical potential is expanded as [43],

$\mu=\mu_{0}+\epsilon^{2} \delta \mu_{2}+\cdots$,

where $\delta \mu_{2}>0$. Thus near the critical point for the order parameter as the function of the chemical potential we have

$\epsilon \approx\left(\frac{\mu-\mu_{0}}{\delta \mu_{2}}\right)^{1 / 2}$,

It is obvious when $\mu \rightarrow \mu_{0}$, the order parameter approaches zero which indicate the phase transition point. Thus phase transition occurs at the critical value $\mu_{c}=\mu_{0}$. Let us note that the order parameter grows with exponent $1 / 2$ which is the universal result from the Ginzburg-Landau mean-field theory.

In the next two sections we solve the field equations (15)-(18) by using the expansions (20)-(23), for the linear Maxwell field as well as the nonlinear power-Maxwell electrodynamics.

\section{Critical temperature of GB holographic superconductors with Maxwell field}

In this section, by using the Sturm-Liouville eigenvalue problem, we obtain the relation between the critical temperature and charge density of the $s$-wave holographic superconductor with backreaction in Gauss-Bonnet-AdS black holes. The Maxwell theory corresponds to $q=1$.

Employing the matching method, the holographic superconductors in Gauss-Bonnet gravity with backreaction for the Maxwell [44] and the nonlinear Born-Infeld electrodynamics have been studied [34,35]. However, it was shown that the matching method is less accurate than SturmLiouville method and the obtained results from SturmLiouville method are in a better agreement with the numerical results.

At zeroth order for the expansion parameter, Eq. (16) may be written as

$\phi_{0}^{\prime \prime}(z)-\frac{\phi_{0}^{\prime}(z)}{z}=0$,

which is the equation of motion of the electromagnetic field in the Maxwell theory and has solution $\phi_{0}(z)=\mu_{0}\left(1-z^{2}\right)$ with $\mu_{0}=\rho / r_{+}^{2}$. At the critical point, we have $\mu_{0}=\mu_{c}=$ $\rho / r_{+c}^{2}$, where $r_{+c}$ is the radius of the horizon at the phase transition point. Therefore, solution of $\phi_{0}(z)$ at the critical point may be written as

$\phi_{0}=r_{+c} \zeta\left(1-z^{2}\right), \quad \zeta \equiv \rho / r_{+c}^{3}$.

Inserting back this solution into Eq. (18), we find the metric function at the zeroth order:

$$
\begin{aligned}
& f_{0}(z)=r_{+}^{2} g(z)=\frac{r_{+}^{2}}{2 \alpha z^{2}} \\
& \quad \times\left(1-\sqrt{1-\frac{4 \alpha}{l^{2}}\left(1-z^{4}\right)+\frac{8 \alpha}{3} \zeta^{2} \kappa^{2} z^{4}\left(1-z^{2}\right)}\right),
\end{aligned}
$$

where we have used the fact that on the horizon $f_{0}(1)=0$, and we have defined a new function $g(z)$ for convenience. We note that $f_{0}(z)$ restores the metric function of GaussBonnet-AdS gravity in the probe limit as $\kappa \rightarrow 0$.

At the first order approximation, the asymptotic AdS boundary conditions for $\psi$ can be expressed as

$\psi_{1} \approx \frac{\psi_{-}}{r_{+}^{-}} z^{\Delta_{-}}+\frac{\psi_{+}}{r_{+}^{\Delta_{+}}} z^{\Delta_{+}}$.

Near the boundary $z=0$, we introduce trial function $F(z)$

$\psi_{1}(z)=\frac{<\mathcal{O}_{i}>}{r_{+}^{\triangle_{i}}} z^{\triangle_{i}} F(z)$,

with boundary condition $F(0)=1$ and $F^{\prime}(0)=0$. Substituting Eqs. (30) into (15) we arrive at

$$
\begin{aligned}
& F^{\prime \prime}(z)+F^{\prime}(z)\left(\frac{g^{\prime}(z)}{g(z)}+\frac{2 \Delta_{i}-1}{z}\right) \\
& +F(z)\left[\frac{\Delta_{i}}{z}\left(\frac{g^{\prime}(z)}{g(z)}+\frac{\Delta_{i}-2}{z}\right)\right. \\
& \left.\times \Delta_{i} z^{2} g(z)^{2}-\frac{m^{2}}{z^{4} g(z)}+\frac{\zeta^{2}\left(z^{2}-1\right)^{2}}{g(z)^{2} z^{4}}\right]=0 .
\end{aligned}
$$

We can convert Eq. (31) into the Standard Sturm-Liouville equation, namely

$\left[T(z) F^{\prime}(z)\right]^{\prime}-Q(z) F(z)+\zeta^{2} P(z) F(z)=0$,

where

$$
\begin{aligned}
Q(z)= & -T(z) \\
& \times\left[\frac{\Delta_{i}}{z}\left(\frac{g^{\prime}(z)}{g(z)}+\frac{\Delta_{i}-2}{z}\right) \Delta_{i} z^{2} g(z)^{2}-\frac{m^{2}}{z^{4} g(z)}\right], \\
P(z)= & T(z) \frac{\left(z^{2}-1\right)^{2}}{g(z)^{2} z^{4}} .
\end{aligned}
$$

According to the Sturm-Liouville eigenvalue problem, $\zeta^{2}$ can be obtained via

$\zeta^{2}=\frac{\int_{0}^{1}\left[T(z)\left[F^{\prime}(z)\right]^{2}+Q(z) F^{2}(z)\right] \mathrm{d} z}{\int_{0}^{1} P(z) F^{2}(z) \mathrm{d} z}$. 
In order to determine $T(z)$ we need to solve equation

$T(z) p(z)=T^{\prime}(z)$

where $p(z)$ is

$p(z)=\left(\frac{g^{\prime}(z)}{g(z)}+\frac{2 \Delta_{i}-1}{z}\right)$.

Since $\alpha$ is small, we can expand the above expression for $p(z)$ and keep terms up to $\mathcal{O}\left(\alpha^{2}\right)$. Then we put the result in Eq. (35) and obtain the following solution for $T(z)$ :

$$
\begin{aligned}
T(z)= & z^{2 \Delta_{i}+1}\left(3\left(z^{-4}-1\right)+2 \zeta^{2} \kappa^{2}\left(z^{2}-1\right)\right) \\
& \times \exp \left\{\left(2+\alpha\left[2 \zeta^{2} \kappa^{2} z^{4}\left(z^{2}-1\right)-3 z^{4}+6\right]\right)\right. \\
& \left.\times \frac{\alpha z^{4}}{6}\left(2 \zeta^{2} \kappa^{2}\left(z^{2}-1\right)-3\right)\right\}
\end{aligned}
$$

For small backreaction parameter, $\kappa$, the explicit expressions for $T(z), Q(z)$ and $P(z)$ up to second order terms of $\alpha$ and $\kappa$, are given by

$$
\begin{aligned}
T(z) \approx & z^{2 \Delta_{i}+1}\left\{3\left(z^{-4}-1\right)+\left[2 \zeta^{2} \kappa^{2}\left(z^{2}-1\right)\right.\right. \\
& \times\left(1+\alpha\left(1+3 \alpha-2(5 \alpha+1) z^{4}+6 \alpha z^{8}\right)\right. \\
& \left.\left.\left.-\alpha\left(z^{2}+1\right)\left(\alpha\left(2 z^{4}-3\right)-1\right)\right)\right]\right\}+\mathcal{O}\left(\alpha^{3}\right) \\
& +\mathcal{O}\left(\kappa^{4}\right), \\
Q(z) \approx & z^{2 \Delta_{i}-5}\left\{3 \Delta _ { i } \left(4+s \Delta_{i} z^{4}-\Delta_{i}\right.\right. \\
& \left.+2 \alpha^{2}\left(\Delta_{i}+8\right) z^{12}-\alpha(5 \alpha+1)\left(\Delta_{i}+4\right) z^{8}\right) \\
& +2 \Delta_{i} \zeta^{2} \kappa^{2} z^{4}\left(6 \alpha^{2} z^{8}\left[\Delta_{i}+8-\left(\Delta_{i}+10\right) z^{2}\right]\right. \\
& -2 \alpha(5 \alpha+1) z^{4}\left[\Delta_{i}+4-\left(\Delta_{i}+6\right) z^{2}\right] \\
& \left.+2 \alpha^{2} \zeta^{2} \kappa^{2} z^{12}\right\}+\mathcal{O}\left(\alpha^{3}\right)+\mathcal{O}\left(\kappa^{4}\right) \\
& \left.\left.+s \Delta_{i}-s\left(\Delta_{i}+2\right) z^{2}+\right)+3 \tilde{m}^{2}\right\}+\mathcal{O}\left(\alpha^{3}\right) \\
& +\mathcal{O}\left(\kappa^{4}\right), \\
& -3 \alpha(\alpha+1)^{2} z^{2 \Delta_{i}-3}\left(z^{2}-1\right)\left\{3\left(\alpha^{2}+2 \alpha-1\right)\right. \\
& \times z^{4}\left[2(1-\alpha) \zeta^{2} \kappa^{2}+3 \alpha(\alpha+1)\right] \\
& +\alpha^{2} z^{8}\left(4 \zeta^{2} \kappa^{2}+3\right)+3 \alpha^{2} z^{10} \\
& \\
& \\
& \\
&
\end{aligned}
$$

where $s=3 \alpha^{2}+\alpha+1$ and hereafter we set $l=1$ for simplicity. In order to use Sturm-Liouville eigenvalue problem, we will use iteration method in the rest of this section. We take $\kappa=\kappa_{n} \Delta \kappa$ where $\Delta \kappa=\kappa_{n+1}-\kappa_{n}$ is step size of iterative procedure and we choose $\Delta \kappa=0.05$. Using the fact that

$\zeta^{2} \kappa^{2}=\zeta^{2} \kappa_{n}^{2}=\left(\left.\zeta^{2}\right|_{\kappa_{n-1}}\right) \kappa_{n}^{2}+\mathcal{O}(\Delta \kappa)^{4}$,

and taking $\kappa_{-1}=\left.\zeta\right|_{\kappa_{-1}}=0$, we obtain the minimum eigenvalue of Eq. (32). We also take the trial function $F(z)=$ $1-a z^{2}$. For example for $\tilde{m}^{2}=-3, \alpha=0.05$ and $\kappa=0$, we have

$\zeta_{\kappa_{0}}^{2}=\frac{-566.794 a^{2}+1096.44 a-737.301}{-7.02708 a^{2}+24.3982 a-26.0408}$,

which attains its minimum $\zeta_{\min }^{2}=19.9456$ for $a=0.7147$. In the second iteration, we take $\kappa=0.05$ and $\left.\zeta^{2}\right|_{\kappa_{0}}=$ 19.9456 in calculation of integrals in Eq. (34), and therefore for $\zeta_{\kappa_{1}}^{2}$, we get

$\zeta_{\kappa_{1}}^{2}=\frac{-559.863 a^{2}+1083.88 a-730.968}{-7.09007 a^{2}+24.5832 a-26.189}$,

which has the minimum value $\zeta_{\min }^{2}=19.7936$ at $a=$ 0.7119 . In Table 1 we summarize our results for $\zeta_{\min }$ and $a$ with different values of Gauss-Bonnet coupling parameter $\alpha$, the backreaction parameter $\kappa$ and reduced mass of scalar field $\tilde{m}^{2}$.

Combining Eqs. (4), (12), (27), and using definition of $\zeta$, we obtain the following expression for the critical temperature:

$T_{c}=\frac{1}{\pi}\left(1-\frac{\kappa^{2} \zeta_{\min }^{2}}{3}\right)\left[\frac{\rho}{\zeta_{\min }}\right]^{1 / 3}$.

We apply the iterative procedure to obtain the critical temperature for different values of $\alpha, \kappa$ and $\tilde{m}^{2}$. In Table 2 we summarize the critical temperature of the phase transition of holographic superconductor in Maxwell electrodynamics for $\Delta_{+}$obtained analytically from Sturm-Liouville method. For comparison, we also provide numerical results which we obtain by using shooting method. In this numerical method we solve Eq. (15) with $\phi(z)$ and $f(z)$ given in Eqs. (27) and (28). Then we find the critical charge density $\rho$ which satisfy the boundary condition $\psi_{-}=0$ in $z \rightarrow 0$. We obtain discrete values of the critical $\rho$ which had this situation. Due to the stability condition [45], we chose the lowest value of $\rho_{c}$ and by using dimensionless quantity $T^{3} / \rho$ we calculated the critical temperature of the phase transition for different values of the Gauss-Bonnet parameter and backreaction parameter.

In order to study the behavior of order parameter with respect to the temperature, we numerically solve field equations (15)-(18) simultaneously by using shooting method. We summarized our results in Fig. 1. In this figure we impose 
Table 1 Analytical results of $\zeta_{\min }^{2}$ and $a$ for Maxwell case with different values of the backreaction $\kappa$ and GB parameter $\alpha$ for $\lambda_{+}$. Here we have taken $\tilde{m}^{2}=-3$

\begin{tabular}{|c|c|c|c|c|c|c|c|c|}
\hline \multirow[t]{2}{*}{$\alpha$} & \multicolumn{2}{|l|}{$\kappa=0$} & \multicolumn{2}{|c|}{$\kappa=0.05$} & \multicolumn{2}{|c|}{$\kappa=0.10$} & \multicolumn{2}{|c|}{$\kappa=0.15$} \\
\hline & $a$ & $\zeta_{\min }^{2}$ & $a$ & $\zeta_{\min }^{2}$ & $a$ & $\zeta_{\min }^{2}$ & $a$ & $\zeta_{\min }^{2}$ \\
\hline-0.19 & 0.7344 & 14.0472 & 0.7330 & 13.9836 & 0.7287 & 13.7949 & 0.7213 & 13.4909 \\
\hline-0.1 & 0.7307 & 15.693 & 0.7290 & 15.6105 & 0.7238 & 15.3662 & 0.7146 & 14.9745 \\
\hline 0 & 0.7218 & 18.2300 & 0.7195 & 18.1097 & 0.7123 & 17.7546 & 0.6996 & 17.1902 \\
\hline 0.1 & 0.7050 & 22.1278 & 0.7015 & 21.9279 & 0.6904 & 21.3407 & 0.6705 & 20.4209 \\
\hline 0.2 & 0.67304 & 28.9837 & 0.6667 & 28.5719 & 0.6462 & 27.3751 & 0.6081 & 25.5561 \\
\hline
\end{tabular}

Table 2 Comparison of analytical and numerical values of the critical temperature for Maxwell case with $\tilde{m}^{2}=-3$

\begin{tabular}{|c|c|c|c|c|c|c|}
\hline \multirow[t]{2}{*}{$\alpha$} & \multicolumn{2}{|l|}{$\kappa=0.05$} & \multicolumn{2}{|l|}{$\kappa=0.10$} & \multicolumn{2}{|l|}{$\kappa=0.15$} \\
\hline & Analytical & Numerical & Analytical & Numerical & Analytical & Numerical \\
\hline-0.19 & $0.2027 \rho^{1 / 3}$ & $0.2050 \rho^{1 / 3}$ & $0.1961 \rho^{1 / 3}$ & $0.1986 \rho^{1 / 3}$ & $0.1854 \rho^{1 / 3}$ & $0.1882 \rho^{1 / 3}$ \\
\hline-0.1 & $0.1987 \rho^{1 / 3}$ & $0.2008 \rho^{1 / 3}$ & $0.1915 \rho^{1 / 3}$ & $0.1938 \rho^{1 / 3}$ & $0.1800 \rho^{1 / 3}$ & $0.1825 \rho^{1 / 3}$ \\
\hline 0 & $0.1935 \rho^{1 / 3}$ & $0.1953 \rho^{1 / 3}$ & $0.1854 \rho^{1 / 3}$ & $0.1874 \rho^{1 / 3}$ & $0.1726 \rho^{1 / 3}$ & $0.1764 \rho^{1 / 3}$ \\
\hline 0.1 & $0.1868 \rho^{1 / 3}$ & $0.1882 \rho^{1 / 3}$ & $0.1775 \rho^{1 / 3}$ & $0.1791 \rho^{1 / 3}$ & $0.1630 \rho^{1 / 3}$ & $0.1646 \rho^{1 / 3}$ \\
\hline 0.2 & $0.1771 \rho^{1 / 3}$ & $0.1779 \rho^{1 / 3}$ & $0.1666 \rho^{1 / 3}$ & $0.1668 \rho^{1 / 3}$ & $0.1499 \rho^{1 / 3}$ & $0.1500 \rho^{1 / 3}$ \\
\hline
\end{tabular}

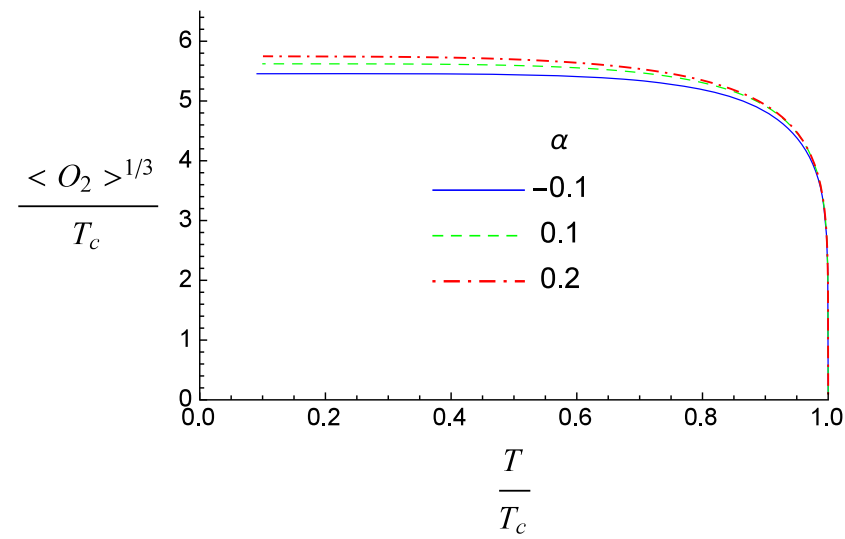

(a) $\kappa=0.05$

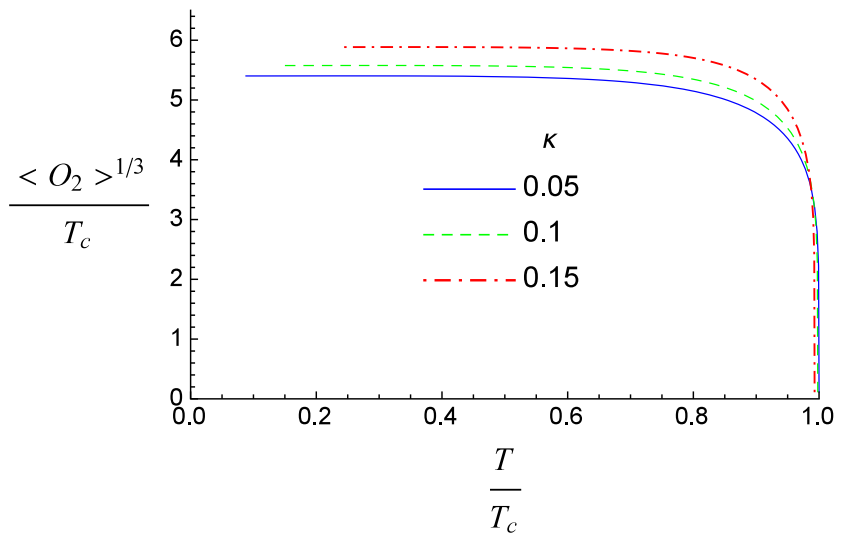

(b) $\alpha=-0.19$

Fig. 1 Condensation as a function of temperature for fixed $\kappa$ and different values of $\alpha$ (a) and for fixed $\alpha$ and different values of $\kappa$ (b). Effective mass of scalar field is $\tilde{m}^{2}=-3$ in both graphs

the boundary condition $\psi_{-}=0$. In Fig. 1a we fixed the backreaction parameter $\kappa$ to see how condensation varies with temperature as we change the Gauss-Bonnet parameter $\alpha$. The temperature is re-scaled to the critical temperature of each case so all the graphs start at point $T / T_{c}=1$. We also fix the Gauss-Bonnet parameter as $\alpha=-0.19$ in Fig. $1 \mathrm{~b}$ and change the backreaction parameter $\kappa$ to show the effects of backreaction on the condensation in all temperatures. Both figures show that the condensation operator increases with increasing $\alpha$ or $\kappa$.

\section{Critical temperature of GB holographic superconductor with power-Maxwell field}

In this section we investigate the behavior of holographic superconductor for the general case $q \neq 1$ away from probe limit in the Gauss-Bonnet gravity. Just like previous section, we need solution of Eqs. (16), (17), and (18) in order to solve (15). Using the expansion (20)-(23) and at the zeroth order of the small parameter $\epsilon$, one can easily check that $\phi_{0}$ and $g$ have the following solution:

$$
\begin{aligned}
& \phi_{0}(z)=\zeta r_{+c}\left(1-z^{\frac{2(2-q)}{2 q-1}}\right), \quad \zeta=\frac{\rho^{\frac{1}{2 q-1}}}{r_{+c}^{\frac{3}{2 q-1}}}, \\
& g(z)=\frac{1}{2 \alpha z^{2}} \\
& \times\left(1-\sqrt{1-4 \alpha\left(1-z^{4}\right)-\frac{4^{q}(2-q)^{2 q-1}}{3(2 q-1)^{2 q-2}} 2 \alpha \kappa^{2} \zeta^{2 q}\left[\left(z^{\frac{6 q}{2 q-1}}\right)-z^{4}\right]}\right),
\end{aligned}
$$


where $g(z)=f_{0}(z) / r_{+}^{2}$. Expanding the above expression for $g(z)$ up to $\mathcal{O}\left(\kappa^{4}\right)$ and $\mathcal{O}\left(\alpha^{2}\right)$, one gets

$$
\begin{aligned}
g(z) \approx & \frac{1}{z^{2}}-z^{2}+\frac{\left(z^{4}-1\right)^{2}}{z^{2}}\left[\alpha-2 \alpha^{2}\left(z^{4}-1\right)\right] \\
& +\frac{(2 q-1)^{2-2 q}(4-2 q)^{2 q-1}\left(z^{\frac{6 q}{2 q-1}}-z^{4}\right)}{3 z^{2}} \\
& \times\left\{\left[6 \alpha^{2}\left(z^{4}-1\right)^{2}-2 \alpha\left(z^{4}-1\right)+1\right\} \kappa^{2} \zeta^{2 q}\right. \\
& -\frac{(2 q-1)^{4-4 q}(4-2 q)^{4 q-2}\left(z^{4}-z^{\frac{6 q}{2 q-1}}\right)^{2}}{9 z^{2}} \\
& \times\left[6 \alpha^{2}\left(z^{4}-1\right)-\alpha\right] \kappa^{4} \zeta^{4 q}+\mathcal{O}\left(\alpha^{3}\right)+\mathcal{O}\left(\kappa^{6}\right) .
\end{aligned}
$$

One may substitute Eqs. (30) and (45) into Eq. (15) and get an expression for $F(z)$, and then converting it to the SturmLiouville equation form (32), results in

$$
\begin{aligned}
& T(z) \approx z^{2 \Delta-3}\left\{1-z^{4}\left[\alpha\left(z^{4}-1\right)\left(\alpha\left(2 z^{4}-3\right)-1\right)\right]\right. \\
& +\frac{(4-2 q)^{2 q}(2 q-1)^{2-2 q}}{6(q-2)} \\
& \times\left[1-z^{\frac{6 q}{2 q-1}}\left(1+\alpha+\alpha^{2}\left(6 z^{8}+3\right)\right)\right. \\
& \left.-\alpha z^{8}\left(1+5 \alpha-4 \alpha z^{4}-2(5 \alpha+1) z^{\frac{4-2 q}{2 q-1}}\right)\right] \kappa^{2} \zeta^{2 q} \\
& +\left[1+\alpha(5 \alpha+1) z^{\frac{12 q}{2 q-1}}+z^{\frac{6 q}{2 q-1}}\right. \\
& \times\left[\alpha\left(\alpha\left(6 z^{8}-3\right)-1\right)-1\right] \\
& \left.-2 \alpha^{2} z^{10}\left(3 z^{\frac{6}{2 q-1}}+z^{2}\right)\right] \kappa^{4} \zeta^{4 q} \\
& \times 2^{4 q}(2-q)^{4 q-2}(1-2 q)^{4-4 q} \\
& 36
\end{aligned}
$$

Again, using Eq. (32), with trial function $F(z)=1-a z^{2}$, we obtain the minimum eigenvalue $\zeta_{\min }^{2}$ for the power-Maxwell electrodynamic case. For example, with $q=3 / 4, \alpha=0.1$, $\kappa=0.05$, and $\tilde{m}^{2}=-3$ and using iterative procedure, we get $\zeta_{\kappa_{1}}^{2}=\frac{30\left(1.0333 a^{2}-2.0068 a+1.3652\right)}{1.3539 a^{2}-4.1680 a+3.6937}$.

Varying $\zeta_{\kappa_{1}}^{2}$ with respect to $a$ to find minimum value of $\zeta^{2}$, we obtain $\zeta_{\min }^{2}=9.50679$ at $a=0.5675$. Also for the case $q=5 / 4, \alpha=-0.19, \kappa=0.1$, and $\tilde{m}^{2}=0$ we obtain

$\zeta_{\kappa_{2}}^{2}=\frac{461.3339 a^{2}-968.8766 a+593.0286}{a^{2}-3.2657 a+3.0859}$,

which attains its minimum $\zeta_{\min }=98.9682$ at $a=0.8909$. Then we find the critical temperature from Eqs. (4), (12), and (45) as

$T_{c}=\frac{1}{4 \pi}\left[4-\frac{(4-2 q)^{2 q}}{3(2 q-1)^{2 q-1}} \kappa^{2} \zeta_{\min }^{2 q}\right]\left(\frac{\rho}{\zeta_{\min }^{2 q-1}}\right)^{\frac{1}{3}}$.

Clearly, $T_{c}$ depends on the power-Maxwell parameter $q$, the Gauss-Bonnet parameter $\alpha$, and the backreaction parameter $\kappa$.

In Fig. 2, we present the reduced critical temperature of the phase transition for a $(3+1)$-dimensional holographic superconductor as a function of $q$ with different values of $\kappa$ and $\alpha$. For simplicity, we focus on the boundary condition which $\psi_{-}=0$, and as an example, we take $\tilde{m}^{2}=-3$ in this figure.

In Fig. 2a we fix the backreaction parameter to $\kappa=0.05$ in order to investigate behavior of the critical temperature as a function of power parameter $q$ for three allowed value of the Gauss-Bonnet parameter. It clearly indicates that, for any values of $\alpha$, by decreasing $q$, superconductor phase is more accessible. Also, we find that in the presence of backreaction of the matter fields on the metric, increasing Gauss-Bonnet parameter $\alpha$ makes condensation harder and thus the critical temperature of the phase transition decreases. It is interesting that decreasing $\alpha$ from zero to negative values in the allowed range can cause the phase transition to superconductor phase easier for any values of the power parameter $q$.

We also provide Fig. 2b by fixing the Gauss-Bonnet parameter to $\alpha=0.1$ for studying the behavior of the reduced critical temperature in terms of the power parameter $q$ for different values of the backreaction parameter $\kappa$. From this figure we see that, for any values of $q$, by increasing the backreaction of the matter fields on the background geometry, which corresponds to decreasing the charge of the scalar field, the phase transition is made harder in the EinsteinGauss-Bonnet gravity.

We mention that in the allowed range of the power parameter, there exist some un-physical regimes in which the critical temperature becomes negative. For example, by increasing the backreaction parameter to greater values, we may obtain a negative $T_{c}$, which means for some values of the power parameter that we do not have a phase transition if a complex field charge is less than some critical charge. Here we 


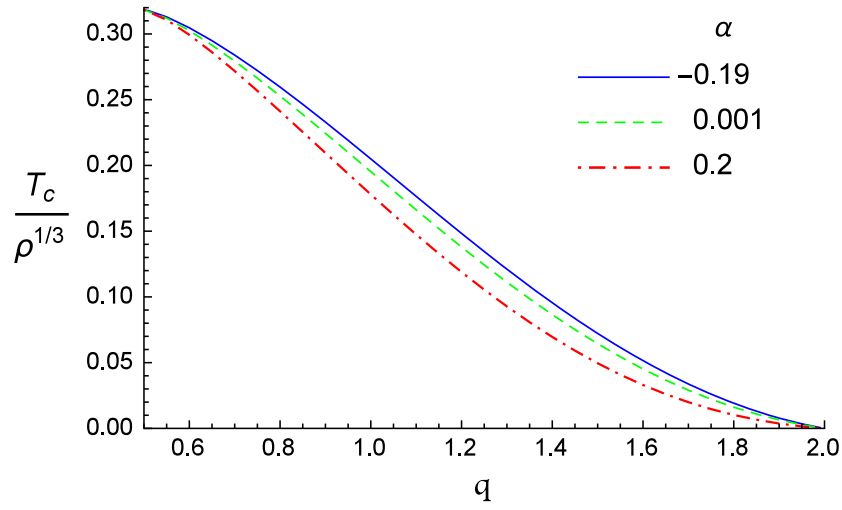

(a) $\kappa=0.05$

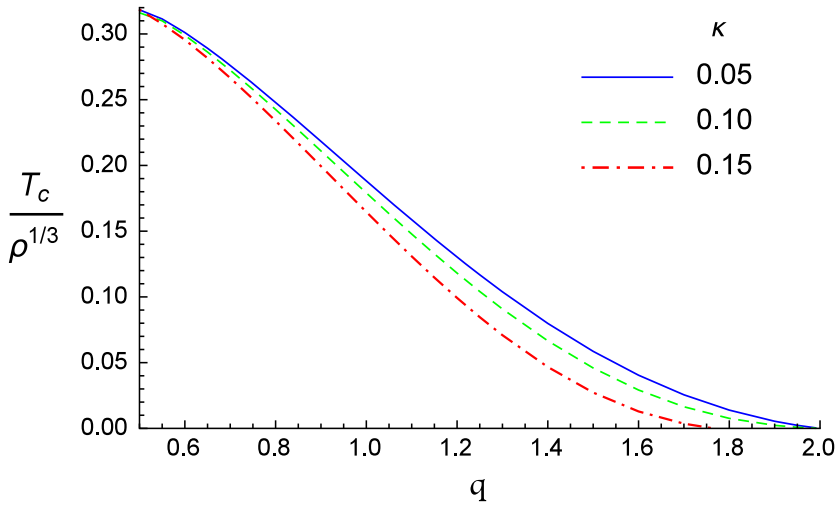

(b) $\alpha=0.10$

Fig. 2 Critical temperature of the GB holographic superconducting phase transition with power-Maxwell field as a function of $q$ for $\tilde{m}^{2}=-3$

Table 3 Comparison of analytical and numerical values of the critical temperature for $\tilde{m}^{2}=-3$ for certain values of $\kappa$ and $\alpha$

\begin{tabular}{|c|c|c|c|c|c|c|}
\hline \multirow[t]{2}{*}{$q$} & \multicolumn{2}{|c|}{$\kappa=0.05, \alpha=0.10$} & \multicolumn{2}{|c|}{$\kappa=0.05, \alpha=-0.01$} & \multicolumn{2}{|c|}{$\kappa=0.10, \alpha=-0.05$} \\
\hline & Analytical & Numerical & Analytical & Numerical & Analytical & Numerical \\
\hline $3 / 4$ & $0.2622 \rho^{1 / 3}$ & $0.2623 \rho^{1 / 3}$ & $0.2666 \rho^{1 / 3}$ & $0.2667 \rho^{1 / 3}$ & $0.2639 \rho^{1 / 3}$ & $0.2642 \rho^{1 / 3}$ \\
\hline 1 & $0.1868 \rho^{1 / 3}$ & $0.1882 \rho^{1 / 3}$ & $0.1940 \rho^{1 / 3}$ & $0.1959 \rho^{1 / 3}$ & $0.1879 \rho^{1 / 3}$ & $0.1908 \rho^{1 / 3}$ \\
\hline $5 / 4$ & $0.1134 \rho^{1 / 3}$ & $0.1168 \rho^{1 / 3}$ & $0.1208 \rho^{1 / 3}$ & $0.1250 \rho^{1 / 3}$ & $0.1124 \rho^{1 / 3}$ & $0.1177 \rho^{1 / 3}$ \\
\hline
\end{tabular}

disregard these regimes and work in regimes with positive temperatures.

Finally, we present Table 3 to compare the results of the critical temperature from analytical Sturm-Liouville method by using iterative procedure with numerical values which we established numerically by using shooting method as explained in previous section. We take different values of $\alpha$ and $\kappa$ in this table for three values of $q$ as example.

\section{Critical exponent}

In this section, we propose to analytically calculate the critical exponent of the Gauss-Bonnet holographic superconductor with backreaction in the general power-Maxwell electrodynamics case for all allowed values of $q$. While we are near the critical point, $\left\langle\mathcal{O}_{i}>\right.$ is small enough, thus we substitute Eq. (30) into the Eq. (16) and by using the fact that in the expansion of $\chi \mathrm{Eq}$. (21) the first term is proportional to $\left\langle\mathcal{O}_{i}\right\rangle^{2}$, while we are near the critical temperature we neglect $\chi^{\prime}(z)$ and arrive at

$$
\begin{aligned}
\phi^{\prime \prime}-\left(\frac{5-4 q}{2 q-1}\right) \frac{1}{z} \phi^{\prime} \\
-\frac{2 r_{+}^{2 q-2 \Delta_{i}-2} z^{2 \Delta_{i}-4 q} F^{2} \phi^{\prime 2-2 q} \phi<\mathcal{O}_{i}>^{2}}{(-1)^{2 q} q(2 q-1) g(z)}=0,
\end{aligned}
$$

where $g(z)$ is defined as in Eq. (47). Near the critical point, $T_{c} \approx T_{0}$, and inspired by Eq. (45), we assume that Eq. (54) obeys the following solution:

$$
\begin{aligned}
\phi(z)= & A T_{c}\left(1-z^{\frac{4-2 q}{2 q-1}}\right)-\left(A T_{c}\right)^{3-2 q} \\
& \times\left(\frac{r_{+}^{2 q-2 \Delta_{i}-2}<\mathcal{O}_{i}>^{2}}{(-1)^{2 q} q(2 q-1)}\right) \Xi(z),
\end{aligned}
$$

where

$$
A=\frac{4 \pi \zeta_{\min }}{4-\frac{(4-2 q)^{2 q}}{3(2 q-1)^{2 q-1}} \kappa^{2} \zeta_{\min }^{2 q}} .
$$

Substituting Eqs. (55) into (54) and keeping terms up to $<\mathcal{O}_{i}>^{2}$, we reach

$$
\begin{aligned}
& \Xi^{\prime \prime}-\left(\frac{5-4 q}{2 q-1}\right) \frac{\Xi^{\prime}}{z} \\
& -\frac{\left(\frac{2 q-4}{2 q-1}\right)^{2-2 q} z^{\eta}\left(1-z^{\frac{4-2 q}{2 q-1}}\right) F(z)^{2}}{g(z)}=0,
\end{aligned}
$$

where

$\eta=2 \Delta_{i}-4 q+\left(\frac{5-4 q}{2 q-1}\right)(2-2 q)$.

This is a differential equation for $\Xi(z)$ independent of $r_{+}, r_{+}$ and $<\mathcal{O}_{i}>$. Therefore $\Xi(z)$ in any $z$ has a value independent of $T, T_{c}$, and order parameter $<\mathcal{O}_{i}>$.

The boundary condition for $\phi$ given by Eq. (13), in the $z$ coordinate, can be rewritten as

$\phi(z)=\mu\left(1-\frac{\rho^{\frac{1}{2 q-1}}}{\mu r_{+}^{\frac{4-2 q}{2 q-1}}} z^{\frac{4-2 q}{2 q-1}}\right)$. 
It is reliable while $z \approx 0$, independent of temperature and order parameter. Also near the critical temperature where $\psi$ is small, Eq. (45) may be expressed as

$\phi_{0}(z)=\frac{\rho^{\frac{1}{2 q-1}}}{r_{+}^{\frac{3}{2 q-1}}} r_{+}\left(1-z^{\frac{4-2 q}{2 q-1}}\right)$,

Since it is valid for all values of $z$, we can equate the above expression with Eq. (59) for $z \rightarrow 0$ to find

$\mu=\frac{\rho^{\frac{1}{2 q-1}}}{r_{+}^{\frac{3}{2 q-1}-1}}$,

Since Eq. (59) implies that, at infinite boundary $z=0$, the gauge field is equal to the chemical potential, i.e., $\phi(z=$ $0)=\mu$. From Eqs. (4) and (12), we realize that $r_{+} \propto T$ and it is obvious from Eq. (53) that $\rho \propto T_{c}^{3}$. Thus by using Eq. (61), one can find

$\phi(z=0)=\mu=A \frac{T_{c}^{\frac{3}{2 q-1}}}{T^{\frac{4-2 q}{2 q-1}}}$.

Equation (55) at $z=0$ is equal to its infinite boundary value given in Eq. (62). Equating Eqs. (55) and (62), we find

$A T_{c}-A \frac{T_{c}^{\frac{3}{2 q-1}}}{T^{\frac{4-2 q}{2 q-1}}}=\left(A T_{c}\right)^{3-2 q}\left(\frac{r_{+}^{2 q-2 \Delta_{i}-2}<\mathcal{O}_{i}>^{2}}{(-1)^{2 q} q(2 q-1)}\right) \Xi(0)$,

where $\Xi(0)$ is just a constant which can be calculated numerically from Eq. (57) with boundary conditions $\Xi(1)=$ $\Xi^{\prime}(1)=1$. Using Eqs. (4) and (12) for replacing $r_{+}$with $T$ in Eq. (63) and then solving the resulting equation for $<\mathcal{O}_{i}>$, we get

$$
\begin{aligned}
<\mathcal{O}_{i}>= & \gamma T_{c}^{\Delta_{i}}\left(\frac{T}{T_{c}}\right)^{\Delta_{i}-q+1} \\
& \times \sqrt{\left(\frac{T_{c}}{T}\right)^{\frac{4-2 q}{2 q-1}}\left[1-\left(\frac{T}{T_{c}}\right)^{\frac{4-2 q}{2 q-1}}\right]},
\end{aligned}
$$

where $\gamma$ is a constant independent of $T$ and $T_{c}$. Using the fact that $T \approx T_{c}$, we can rewrite $<\mathcal{O}_{i}>$ as

$$
\begin{aligned}
& <\mathcal{O}_{i}>\approx \gamma T_{c}^{\Delta_{i}} \sqrt{1-\left(\frac{T}{T_{c}}\right)^{\frac{4-2 q}{2 q-1}}} \\
& \approx \gamma T_{c}^{\Delta_{i}} \sqrt{1-\left[1-\left(\frac{4-2 q}{2 q-1}\right) t\right]} \approx \gamma T_{c}^{\Delta_{i}} \sqrt{\left(\frac{4-2 q}{2 q-1}\right) t},
\end{aligned}
$$

where $t=\left(T_{c}-T\right) / T_{c}$. Equation (65) indicates that the critical exponent $\beta$ of the order parameter is $1 / 2$ and this result is valid both for $\left\langle\mathcal{O}_{-}>\right.$and $<\mathcal{O}_{+}>$. It is obvious that in the presence of backreaction this exponent for Gauss-Bonnet gravity with power-Maxwell field remains unchanged which seems to be a universal exponent. Let us note that, for $q=2$, the expectation value of the condensation operator vanishes, which means there is no phase transition in the upper bound of $q$.

\section{Conclusion and discussion}

Analytically and based on Sturm-Liouville eigenvalue problem, we have investigated the properties of $(3+1)$ dimensional $s$-wave holographic superconductors in the background of five-dimensional Gauss-Bonnet-AdS black holes with power-Maxwell electrodynamics. We have considered the case in which the gauge and scalar fields back react on the background geometry. We find the relation between the critical temperature of the phase transition and the charge density is still $T_{c} \propto \rho^{1 / 3}$. Using the analytical Sturm-Liouville method, we have calculated the proportional constant between the critical temperature and the charge density for all allowed values of the power parameter $q$, different values of the Gauss-Bonnet coupling constant $\alpha$, and of the backreaction parameter $\kappa$. We realized that decreasing $q$ from the Maxwell case $(q=1)$ to its lower bound $(q=1 / 2)$ increases the critical temperature, regardless of the values of $\alpha$ and $\kappa$. Besides, for a fixed value of $q$ and $\kappa$, the critical temperature increases with decreasing Gauss-Bonnet coefficient $\alpha$. This means that increasing $q$ and $\alpha$ will decrease the critical condensation of the scalar field and make it harder to form. Also, we observed that taking backreaction into account decreases the critical temperature regardless of the values of the other parameters. We have confirmed these analytical results by providing the numerical calculations based on the shooting method. Finally, our investigation of the critical exponent indicates that the critical exponent $\beta$ of the superconducting phase transition for the five-dimensional powerMaxwell holographic superconductor with backreaction has the mean-field value $1 / 2$, which seems to be a universal constant.

Acknowledgements We thank the referee for constructive comments which helped us improve the paper significantly. We also thank Shiraz University Research Council. The work of A.S has been supported financially by Research Institute for Astronomy and Astrophysics of Maragha (RIAAM), Iran.

Open Access This article is distributed under the terms of the Creative Commons Attribution 4.0 International License (http://creativecomm ons.org/licenses/by/4.0/), which permits unrestricted use, distribution, and reproduction in any medium, provided you give appropriate credit to the original author(s) and the source, provide a link to the Creative Commons license, and indicate if changes were made. Funded by SCOAP $^{3}$. 


\section{References}

1. S.A. Hartnoll, C.P. Herzog, G.T. Horowitz, Building a holographic superconductor. Phys. Rev. Lett. 101, 031601 (2008). arXiv:0803.3295

2. S.A. Hartnoll, C.P. Herzog, G.T. Horowitz, Holographic superconductors. JHEP 12, 015 (2008). arXiv:0810.1563

3. J. Maldacena, The large $\mathrm{N}$ limit of superconformal field theories and supergravity. Adv. Theor. Math. Phys. 2, 231 (1998). arXiv:hep-th/9711200

4. S.S. Gubser, I.R. Klebanov, A.M. Polyakov, Gauge theory correlators from non-critical string theory. Phys. Lett. B 428, 105 (1998). arXiv:hep-th/9802109

5. E. Witten, Anti de sitter space and holography. Adv. Theor. Math. Phys. 2, 253 (1998). arXiv:hep-th/9802150

6. S.S. Gubser, Breaking an Abelian gauge symmetry near a black hole horizon. Phys. Rev. D 78, 065034 (2008). arXiv:0801.2977

7. G.T. Horowitz, Introduction to holographic superconductors. Lect. Notes Phys. 828, 313 (2011)

8. D. Musso, Introductory notes on holographic superconductors. arXiv: 1401.1504

9. R.G. Cai, L. Li, L.-F. Li, R.-Q. Yang, Introduction to holographic superconductor models. Sci. China Phys. Mech. Astron. 58, 060401 (2015). arXiv: 1502.00437

10. X.H. Ge, B. Wang, S.F. Wu, G.H. Yang, Analytical study on holographic superconductors in external magnetic field. JHEP 08, 108 (2010). arXiv:1002.4901

11. R. Banerjee, S. Gangopadhyay, D. Roychowdhury, A. Lala, Holographic s-wave condensate with non-linear electrodynamics: a nontrivial boundary value problem. Phys. Rev. D 87, 104001 (2013). arXiv: $1208.5902 \mathrm{v} 3$

12. D. Momeni, M. Raza, R. Myrzakulov, More on superconductors via gauge/gravity duality with nonlinear Maxwell field. J. Gravity 2013, Article ID 782512 (2013)

13. C.M. Chen, M.F. Wu, An analytic analysis of phase transitions in holographic superconductors. Prog. Theor. Phys. 126, 387 (2011). arXiv: 1103.5130

14. H.B. Zeng, X. Gao, Y. Jiang, H.S. Zong, Analytical computation of critical exponents in several holographic superconductors. JHEP 1105, 002 (2011). arXiv: 1012.5564

15. R.G. Cai, H.F. Li, H.Q. Zhang, Analytical studies on holographic insulator/superconductor phase transitions. Phys. Rev. D 83, 126007 (2011). arXiv:1103.5568

16. R.G. Cai, L. Li, L.F. Li, A holographic p-wave superconductor model. JHEP 1401, 032 (2014). arXiv:1309.4877

17. Q. Pan, B. Wang, E. Papantonopoulos, J. Oliveira, A.B. Pavan, Holographic superconductors with various condensates in Einstein-Gauss-Bonnet gravity. Phys. Rev. D 81, 106007 (2010). arXiv:0912.2475

18. Q. Pan, B. Wang, General holographic superconductor models with Gauss-Bonnet corrections. Phys. Lett. B 693, 159 (2010). arXiv: 1005.4743

19. H.F. Li, R.G. Cai, H.Q. Zhang, Analytical studies on holographic superconductors in Gauss-Bonnet gravity. JHEP 04, 028 (2011). arXiv: 1103.2833

20. L. Barclay, R. Gregory, S. Kanno, P. Sutcliffe, GaussBonnet holographic superconductors. JHEP 1012, 029 (2010). arXiv:1009.1991

21. R.G. Cai, Z.Y. Nie, H.Q. Zhang, Holographic p-wave superconductors from Gauss-Bonnet gravity. Phys. Rev. D 82, 066007 (2010). arXiv: 1007.3321

22. R.G. Cai, Z.Y.Nie, H.Q. Zhang, Holographic phase transitions of Pwave superconductors in Gauss-Bonnet gravity with back-reaction. Phys. Rev. D 83, 066013 (2011). arXiv:1012.5559
23. Q. Pan, J. Jing, B. Wang, Analytical investigation of the phase transition between holographic insulator and superconductor in GaussBonnet gravity. JHEP 11, 088 (2011). arXiv:1105.6153

24. Z. Zhao, Q. Pan, S. Chen, J. Jing, Notes on holographic superconductor models with the nonlinear electrodynamics. Nucl. Phys. B 871, 98 (2013). arXiv: 1212.6693

25. J. Jing, Q. Pan, S. Chen, Holographic superconductor/insulator transition with logarithmic electromagnetic field in Gauss-Bonnet gravity. Phys. Lett. B 716, 385 (2012). arXiv:1209.0893

26. A. Sheykhi, F. Shaker, Effects of backreaction and exponential nonlinear electrodynamics on the holographic superconductors. arXiv:1606.04364

27. A. Sheykhi, F. Shamsi, Holographic superconductors with logarithmic nonlinear electrodynamics in an external magnetic field. arXiv: 1603.02678

28. C. Lai, Q. Pan, J. Jing, Y. Wang, On analytical study of holographic superconductors with Born-Infeld electrodynamics. Phys. Lett. B 749, 437 (2015). arXiv: 1508.05926

29. D. Ghorai, S. Gangopadhyay, Analytic study of higher dimensional holographic superconductors in Born-Infeld electrodynamics away from the probe limit. arXiv:1511.02444

30. P. Chaturvedi, G. Sengupta, p-wave holographic superconductors from Born-Infeld black holes. JHEP 1504, 001 (2015). arXiv: 1501.06998

31. S. Gangopadhyay, D. Roychowdhury, Analytic study of properties of holographic superconductors in Born-Infeld electrodynamics. JHEP 05, 156 (2012). arXiv: 1201.6520

32. A. Sheykhi, F. Shaker, Analytical study of holographic superconductor in Born-Infeld electrodynamics with backreaction. Phys. Lett. B 754, 281 (2016). arXiv:1601.04035

33. G. Siopsis, J. Therrien, Analytic calculation of properties of holographic superconductors. JHEP 05, 013 (2010). arXiv:1003.4275

34. W. Yao, J. Jing, Analytical study on holographic superconductors for Born-Infeld electrodynamics in Gauss-Bonnet gravity with backreactions. JHEP 05, 101 (2013). arXiv:1306.0064

35. J. Jing, L. Wang, Q. Pan, S. Chen, Holographic Superconductors in Gauss-Bonnet gravity with Born-Infeld electrodynamics. Phys. Rev. D 83, 066010 (2011). arXiv:1012.0644

36. S. Dey, A. Lala, Holographic s-wave condensation and Meissnerlike effect in Gauss-Bonnet gravity with various non-linear corrections. Ann. Phys. 354, 165 (2015). arXiv: 1306.5137

37. R. Gregory, S. Kanno, J. Soda, Holographic superconductors with higher curvature corrections. JHEP 0910, 010 (2009). arXiv:0907.3203

38. J. Jing, Q. Pan, S. Chen, Holographic superconductors with PowerMaxwell field. JHEP 11, 045 (2011). arXiv:1106.5181

39. J. Jing, L. Jiang, Q. Pan, Holographic superconductors for the Power-Maxwell field with backreactions. Class. Quantum Grav. 33, 025001 (2016)

40. A. Sheykhi, H.R. Salahi, A. Montakhab, Analytical and numerical study of Gauss-Bonnet holographic superconductors with PowerMaxwell field. JHEP 04, 058 (2016)

41. M. Hassaine, C. Martinez, Higher-dimensional black holes with a conformally invariant Maxwell source. Phys. Rev. D 75, 027502 (2007)

42. M. Hassaine, C. Martinez, Higher-dimensional charged black holes solutions with a nonlinear electrodynamics source. Class. Quant. Grav. 25, 195023 (2008)

43. C.P. Herzog, Analytic holographic superconductor. Phys. Rev. D 81, 126009 (2010). arXiv:1003.3278 [hep-th]

44. S. Kanno, A note on Gauss-Bonnet holographic superconductors. Class. Quant. Grav. 28, 127001 (2011). arXiv:1103.5022

45. S.S. Gubser, S.S. Pufu, The gravity dual of a p-wave superconductor. JHEP 11, 033 (2008). arXiv:0805.2960 